\title{
Multiple determinants of "blocking" effects on operant behavior
}

\author{
BEN A. WILLIAMS and NICHOLAS HEYNEMAN \\ University of California, San Diego, La Jolla, California 92093
}

\begin{abstract}
Blocking was investigated in a free-operant procedure by presenting a response-contingent signal prior to reinforcer delivery. At issue was the way in which blocking effects previously reported with this procedure are related to conditioned reinforcement effects, also previously found with similar procedures. Signal presentation decreased response rate when delay of reinforcement was 0 or $.5 \mathrm{sec}$, but the signal increased response rate when the delay of reinforcement was increased to $3 \mathrm{sec}$. Thus, which effect (blocking or conditioned reinforcement) occurred depended critically on the response-reinforcer interval.
\end{abstract}

A variety of studies have extended the blocking effect (cf. Kamin, 1969) to instrumental conditioning (Mackintosh \& Dickinson, 1979; Pearce \& Hall, 1978; St. Claire-Smith, 1979a, 1979b; Williams, 1975, 1978, in press; Zanich \& Fowler, 1978). The general plan of all of these studies was to present a stimulus, previously paired with the reinforcer, immediately preceding the reinforcer produced by the instrumental response. The expectation has been that the prior existence of the stimulus-reinforcer association should compete with the acquisition of the response-reinforcer association and thus reduce the instrumental behavior. In other words, the occurrence of the stimulus predicts the occurrence of the reinforcer, so less learning about the response-reinforcer association is engendered.

A major problem for the interpretation of the above studies is the difficulty in disentangling responsereinforcer associations from associations involving the discriminative stimulus for the response. Many of the above studies have used discrete-trial procedures, in which some type of stimulus is differentially correlated with the availability of reinforcement. Thus, it is possible that the blocking manipulation has its effects by changing the role played by that discriminative stimulus. For example, Mackintosh \& Dickinson (1979) paired a tone stimulus with a running-wheel response that was intermittently available (the wheel could be locked to prevent running). When the tone was positively as-

Requests for reprints should be sent to the first author, Department of Psychology, C-009, University of California, San Diego, La Jolla, California 92093. The second author is now at the University of West Virginia. The present study was previously reported at the 1979 meeting of the Psychonomic Society. Preparation of the manuscript was supported by NIMH Research Grant ROI MH-35572-01. The authors thank E. J. Fantino and A. Dickinson for their helpful comments on earlier versions of the manuscript. sociated with food on trials not involving running, the effect of food reinforcement on running was reduced (measured on test trials without the tone); when the tone was negatively associated with food on additional trials, the strength of the running response was enhanced. The interpretative difficulty with this procedure is that a variety of cues (e.g., the unlocking of the wheel, the movement of the wheel as feedback) were differentially associated with the availability of running, so that the animal should have learned about the relation between those cues and when running could be reinforced. The tone should compete with such "trial" cues for stimulus control, and it is likely that the effectiveness of that competition should depend on the tone's associational history. Thus, the rate of running on test trials might reflect only the power of the trial cues to initiate running rather than changes in the strength of the running response per se.

An alternative method for dissociating the effects of stimulus control from those of the responsereinforcer association has been to use a free-operant procedure in which the stimulus situation remains constant throughout the experimental session. Thus, no particular stimulus is correlated with the delivery of food, which is predicted entirely by the occurrence of the instrumental response. The procedure is illustrated by Pearce and Hall (1978), who trained two groups of rats on variable-interval (VI) schedules of food reinforcement. One group received responsedependent food always correlated with a brief stimulus; the second group received response-dependent food but with the same stimulus presented randomly throughout the session. In one experiment, a .5-sec delay was interposed between the response and food; in a second experiment, food was presented immediately after the response. For both experiments, Pearce and Hall found less responding in the correlated-stimulus group than in the group with the 
randomly arranged stimulus. They suggested that the stimulus was a better predictor of food than the response and thus served to attenuate the responsefood association. Assuming that changes in the degree of stimulus control cannot be the mediator of the effect, the apparent implication is that responsereinforcer and stimulus-reinforcer associations are indeed in competition.

While the results of Pearce and Hall (1978) are consistent with other studies of blocking in instrumental learning situations, they are puzzling when viewed in the context of the general nature of freeoperant procedures. Whereas they explicitly programmed the stimulus that was correlated with the occurrence of the reinforcer, operant procedures generally include such stimuli even when not explicitly programmed. For example, the click of the food magazine, the onset of the food-hopper light, and even the presentation of the food itself could be viewed as stimuli that predict reward, since they are presented only in conjunction with it. Thus, in virtually all operant procedures, some stimuli other than the response signal the reinforcer and, presumably, according to the notion of blocking, should serve to reduce the response-reinforcer association. The issue raised is why the addition of one more signal by the experimenter should be of importance. In other words, why should the difference between one vs. two signals or two vs. three, depending on how such stimuli are counted, be of so much importance in the determination of blocking?

The results of Pearce and Hall (and of comparable experiments, e.g., St Claire-Smith, 1979a, 1979b) are also surprising when viewed in the context of previous work on conditioned reinforcement in freeoperant procedures. Inherent in the concept of conditioned reinforcement is that a response-dependent stimulus, previously paired with food, will facilitate instrumental behavior. That is, the stimulus has conditioned reinforcement properties by virtue of its pairing with food. Such properties are readily apparent in previous studies of delay of reinforcement, which are at least conceptually similar to the recent blocking studies. If an unsignaled delay is imposed between a response and a contingent reinforcer, responding will decrease substantially (Williams, 1976). However, presentation of a stimulus during the delay interval will counteract the detrimental effects of the delay and will maintain responding at a rate comparable to that when reinforcement is presented immediately (Pierce, Hanford, \& Zimmerman, 1972; Richards, 1972; Richards \& Hittesdorf, 1978).

The issue is how a stimulus paired with reward may affect behavior in two diametrically opposite ways: strengthening behavior because of its conditioned reinforcement properties, but weakening behavior by attenuating the response-reinforcer asso- ciation. Clearly, therefore, the relation between previous work on conditioned reinforcement and the more recent work on blocking warrants closer consideration.

One obvious difference between blocking studies and conditioned reinforcement studies has been the different delay values imposed between the response and reinforcer. Blocking studies have used minimal delay values (less than $1 \mathrm{sec}$ ), while conditioned reinforcement studies typically have used much longer delays. The present study was an investigation of the delay interval to ascertain whether it is a critical parameter. To do this, responding was maintained on a multiple VI VI schedule of food reinforcement. During one component of the multiple schedule, an added stimulus was made contingent upon the reinforced response. During the alternate component, no signal was added. Both components had the same schedule value. A delay-of-reinforcement contingency was imposed consisting of three conditions: (1) 0 delay (food immediate in both components, with a .5-sec signal coincident with food in one component); (2) .5 -sec delay (food delayed $.5 \mathrm{sec}$ in both components and a .5-sec signal during the delay in one component); (3) 3-sec delay (food delayed $3 \mathrm{sec}$ in both components and a 3 -sec signal during the delay in one component).

On the basis of recent studies of blocking, a decrement in responding in the signaled component, compared with the unsignaled component, would be expected. On the basis of the conditioned reinforcement literature, no decrease in responding in the signaled component would be expected, but a decrement would be expected during the unsignaled component as the delay interval is increased.

\section{METHOD}

\section{Subjects}

Four White Carneaux pigeons were maintained at $80 \%$ of their free-feeding body weights. Two were experimentally naive (G-13, $R-60$ ), while two had had previous experience with schedules of food reinforcement.

\begin{abstract}
Apparatus
A standard single-key pigeon conditioning chamber was constructed from a plastic picnic chest. The pigeon's chamber was $30.5 \mathrm{~cm}$ in all dimensions. A transparent pigeon $\mathrm{key}, 1.6 \mathrm{~cm}$ in diameter and requiring a force of at least $.10-.12 \mathrm{~N}$ for operation, was mounted on the front panel. The stimuli were projected on the rear of the key by a standard 28-V dc 12-stimulus IEE in-line projector. Ten centimeters below the key was a $5 \times 5 \mathrm{~cm}$ aperture through which the birds were fed when the grain magazine was operated. Located on the rear wall of the inner chamber was a $28-\mathrm{V}$ dc houselight that served as the signal and was illuminated only at programmed intervals. Programming and recording equipment were located in an adjacent room.
\end{abstract}

\section{Procedure}

The two naive subjects were trained to eat from the food hopper and were then autoshaped to peck the response key. All 
subjects were given four training sessions before the start of the delay conditions. During the first session, a multiple VI 20-sec VI 20 -sec schedule of food reinforcement was presented. One component was signaled by a blue keylight, while the other component was signaled by a yellow keylight. Components were alternated, and for this session each subject received two exposures to each component. Component duration for this and all subsequent sessions was $5 \mathrm{~min}$. The second and third sessions were identical to the first except that each subject received three exposures to each of the two components. During the fourth session, and for all following sessions, all subjects received eight exposures to each of the two components, making the total session length $80 \mathrm{~min}$. For this and all following sessions, a multiple VI 3-min VI 3-min schedule of reinforcement was in effect. Reinforcement consisted of 3-sec access to mixed grain, during which the keylight remained illuminated. For the first four sessions, the houselight was never illuminated.

After preliminary training, the subjects were exposed to the houselight signal during one component and not during the alternate component. Two subjects received the blue keylight as the discriminative stimulus for the signaled component and the yellow keylight as the discriminative stimulus for the unsignaled component. For the remaining two subjects, the keylight assignments were reversed.

Three different delay values were presented, with the same delays always used during both components of the schedule. Initially, all subjects were presented a 0 delay (immediate reinforcement). For the signaled component, this meant that the signal, a .5-sec illumination of the houselight, was presented coincidently with the hopper presentation. After 30 sessions of training with a 0 delay, a .5-sec delay was imposed during both components. For the signaled component, a .5-sec illumination of the houselight was presented during the delay interval and was terminated with the delivery of the reinforcer. During the unsignaled component, there was no signal during the delay since the keylight remained on. After 20 sessions of training, the delay value was again extended to $3 \mathrm{sec}$ for 20 additional sessions. For the signaled component, the houselight signal was again illuminated during the entire delay interval and was terminated with the food delivery. For the unsignaled component, no signal was present during the delay, the keylight remained on, and the birds could continue to peck during the delay. Finally, all subjects were returned to the 0 -delay condition for 20 additional sessions, except for Subject G-13, which received 65 additional sessions.

\section{RESULTS}

Figure 1 shows the response rate, averaged over subjects, for the last four sessions of each delay-ofreinforcement condition. Rates were calculated excluding responses during the delay intervals. The connected points represent behavior during the first exposure to each condition. The unconnected points represent the replication of the 0 -delay condition at the end of training. The pattern of responding for individual subjects was entirely consistent with the mean rates, with one minor exception that will be noted later.

Increases in the delay reinforcement clearly had quite different effects in the two components of the schedule. For the signaled component, the delay gradient was relatively flat, with the lowest rate in the 0-delay condition. For the unsignaled component, the delay gradient was clearly nonmonotonic. There was an increase in rate with the change to the $.5-\mathrm{sec}$

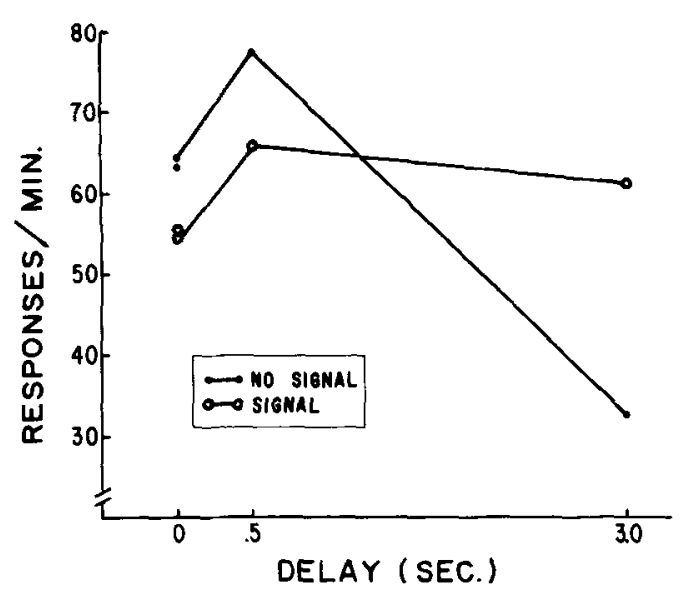

Figure 1. Response rates, averaged over subjects, for the last four sessions of training on each condition. Separate functions are presented for the signaled and unsignaled components of the multiple schedule. The unconnected points correspond to the replication of the 0-delay condition.

delay (consistent for all subjects) and then a sharp decrease with a further increase to $3 \mathrm{sec}$. This meant that the response rates were higher during the unsignaled component for the 0 - and .5-sec delays, but substantially lower than the signaled component for the 3-sec delay.

The results shown in Figure 1 were tested statistically with a two-way analysis of variance (delay $\times$ signaling condition), with a .05 criterion of significance. For the purposes of the test, the results of the two exposures to the 0-delay condition were averaged together for each subject. The effect of the delay variable was significant $[F(2,6)=18.1]$. The effect of the signaling variable was not significant $[F(1,3)=.23]$. The interaction term was also significant $[F(2,6)=20.65]$. Because of the significant interaction, separate tests were conducted on the difference between the signaled and unsignaled components for each delay value. For these tests, the two exposures to the 0 -delay condition were treated separately. All tests had $3 \mathrm{df}$ and used a .05 criterion of significance. For the first exposure to the 0 -delay condition, the difference was statistically significant $(t=3.15)$. The differences were also significant for the .5 -sec delay $(\mathrm{t}=3.21)$ and the $3.0-\mathrm{sec}$ delay $(t=3.43)$. The difference during the second exposure to the 0 -delay condition was not significant $(t=1.44)$. The reason for this is that one subject failed to recover stable responding during the unsignaled component even after considerable additional training (65 sessions). Instead, frequent periods of nonresponding were interspersed with periods of stable responding, with the result that the average rate during the unsignaled component remained lower than the average rate during the signaled component. It is noteworthy that this subject was strongly affected by the 3.0-sec delay of reinforcement in the 
preceding condition, since its response rate during the unsignaled component was reduced by $90 \%-95 \%$ from its previous level. In any event, its failure to recover the original difference between the signaled and unsignaled component was balanced by the remaining three subjects, which all recovered their original patterns of behavior.

\section{DISCUSSION}

The results shown in Figure 1 are consistent both with previous studies of blocking in free-operant procedures (Pearce \& Hall, 1978; St. Claire-Smith, $1979 \mathrm{a}, 1979 \mathrm{~b})$ and with previous studies of conditioned reinforcement (e.g., Pierce et al., 1972, vs. Williams, 1976). With either a 0 delay or .5-sec delay, a "blocking" effect was demonstrated, since lower response rates were maintained when the reinforcer was associated with an exteroceptive signal. With a delay value only slightly longer $(3 \mathrm{sec})$, the blocking effect was replaced by a conditioned reinforcement effect, since a higher rate was maintained when the reinforcer was signaled. Apparently, therefore, the same stimulus can serve two opposite functions, depending upon the delay-of-reinforcement parameter.

The results are also consistent with a recent study of delayed reinforcement that also compared signaled and unsignaled delay procedures, but with the two conditions presented over successive blocks of sessions rather than as alternate components of a multiple schedule (Richards, 1981). As in the present study, Richards found that the unsignaled procedure produced substantially greater response decrements with long delays for all subjects. His results were more variable with the shorter delays $(.5 \mathrm{sec}$ was the shortest delay tested), but for three of the four subjects the unsignaled procedure produced higher response rates. Thus, his results were essentially like the crossover effect seen in Figure 1 of the present study.

Although the results with the short delay values do replicate previous findings of blocking in freeoperant procedures, they also show such effects to be limited to a very small range of parameter values. But, perhaps most important is the possibility that blocking is not the proper interpretation of the difference found with the $.5-\mathrm{sec}$ delay. This possibility is suggested by the increase in response rate during the unsignaled component when the condition was changed from 0 delay to .5-sec delay. Such an increase is similar to previous findings with the unsignaled delay procedure in which the delay gradient has been determined for a larger set of delay values (Richards, 1981; Sizemore \& Lattal, 1978). The nonmonotonic gradient is important to the present discussion because its best interpretation appears to be in terms of changes in response topography that are engendered by very short delays. As argued by Sizemore \& Lattal (1978), short delays favor the reinforcement of response bursts instead of single pecks, because single pecks will receive delayed reinforcement, while bursts will be reinforced immediately (since the first peck of the burst initiates the delay interval and the burst continues throughout the delay). The implication is that the difference between the signaled and unsignaled components with the .5-sec delay may be due not to blocking, but rather to an inherent difference between immediate reinforcement (assuming that the signal is a conditioned reinforcer) and delayed reinforcement in which short delays maintain higher response rates.

Although the effects of delay on response topography provide an adequate explanation of the differences found with the $.5-\mathrm{sec}$ delay, they do not explain the similar differences that were found with the 0-delay condition. The signal occurred simultaneously with the reinforcer in that condition, so the difference between the signaled and unsignaled components cannot be ascribed to the difference between delayed and immediate reinforcement per se. A similar argument was made by Pearce and Hall (1978), who also reported blocking effects with both .5-sec and 0-sec delay values. But the "blocking" effect obtained with the 0-delay condition is highly counterintuitive. Because the signal was coincident with the reinforcer, it was also coincident with the illumination of the hopper light. Any account based on blocking must therefore explain why the hopper light itself did not serve the same function as the houselight signal. Such a difficulty provides strong encouragement for questioning the validity of the blocking explanation for the 0-delay condition as well.

An alternative interpretation assumes that the presence of the houselight signal affected response topography. Because the houselight was correlated with reinforcement, it is possible that it elicited an orienting response before the food hopper was approached. Thus, the pattern of behavior that preceded reinforcement was to peck and orient during the signaled component, but only to peck during the unsignaled component. Such a difference would be expected to decrease the rate of pecking during the signaled component because the response unit would be less efficient.

Support for this interpretation comes from a recent analysis of barpressing behavior of rats under a 2-sec signaled delay procedure (Iverson, 1981). The rats were observed both before and after the signal was added, with the result that the rats ceased responding during the signal but instead spent their time orienting toward it. Furthermore, the pattern of behavior at times other than the signal was also changed, since barpressing was frequently interrupted 
by periods in which the rats oriented toward the signal location. Thus, the overall reduction in response rate that occurred with the signaled delay was ascribed to changes in response topography rather than to a decrease in response strength per se. Such considerations imply that previous reports of "blocking" (or "overshadowing") must be interpreted with caution, because such effects can occur for several different reasons.

\section{REFERENCES}

IVERsEN, I. H. Response interactions with signalled delay of reinforcement. Behoviour Analysis Letters, 1981, 1, 3-9.

Kamin, L. J. Predictability, surprise, attention, and conditioning. In B. Campbell \& R. Church (Eds.), Punishment and aversive behavior. New York: Appleton-Crofts, 1969.

Mackintosh, N. J., \& Dickinson, A. Instrumental (type II) conditioning. In A. Dickinson \& R. Boakes (Eds.), Mechanisms of learning and motivation: $A$ memorial volume to Jerzy Konorski. Hillsdale, N.J: Eslbaum, 1979.

Pearce, J. J., \& Hall., B. Overshadowing of instrumental conditioning of a lever press response by a more valid predictor of reinforcement. Journal of Experimental Psychology: Animal Behavior Processes, 1978, 4, 356-367.

Pierce, C. H., Hanford, P. V., \& Zimmerman, J. Effects of different delay-of-reinforcement procedures on variable-interval responding. Journal of the Experimental Analysis of Behavior, $1972,18,141-146$.

Richands, R. W. Reinforcement delay: Some effects on behavioral contrast. Journal of the Experimental Analysis of Behavior, 1972, 17, 381-394.
Richards, R. W. A comparison of signalled and unsignalled delay of reinforcement. Journal of the Experimental Analysis of Behavior, 1981, 35, 145-152.

Richards, R. W., \& Hitesdonf, W. M. Inhibitory stimuius control under conditions of signalled and unsignalled delay of reinforcement. Psychological Record, 1978, 28, 615-625.

St, Claire-Smith, R. J. The overshadowing and blocking of punishment. Quarterly Journal of Experimental Psychology, $1979,31,51-61$. (a)

St. Claire-Smith, R. J. The overshadowing of instrumental conditioning by a stimulus that predicts reinforcement better than the response. Animal Learning \& Behavior, 1979, 7, 224228. (b)

Sizemore, O. J., \& LatTal, K. A. Unsignalled delay of reinforcement in variable-interval schedules. Journal of the Experimental Analysis of Behavior, 1978, 30, 169-175.

Williams, B. A. The blocking of reinforcement control. Journal of the Experimental Analysis of Behavior, 1975, 24, 215-225.

Wilciams, B. A. The effects of unsignalled delayed reinforcement. Journal of the Experimental Analysis of Behavior, 1976, 26, $441-449$.

Wiltiams, B. A. Information effects on the response-reinforcer association. Animal Learning \& Behavior, 1978, 6, 371-379.

Wilusams, B. A. Blocking the response-reinforcer association. In M. Commons, A. Wagner, \& R. J. Herrnstein (Eds.), Harvard Symposium on Quantitative Analyses of Behavior: Acquisition. Cambridge, Mass: Ballinger Press, in press.

Zanich, M. L., \& Fowler, H. Transfer from Pavlovian appetitive to instrumental appetitive conditioning: Signalling versus discrepancy interpretations. Journal of Experimental Psychology: Animal Behavior Processes, 1978, 4, 37-49.

(Manuscript received July 16, 1981; revision accepted for publication October 20,1981 .) 